\title{
Physics conditions for robust control of tearing modes in a rotating tokamak plasma
}

E. Lazzaro ${ }^{1}$, D. Borgogno ${ }^{3}$, D. Brunetti ${ }^{1}$, L. Comisso $^{4}$, O. Fevrier ${ }^{5}$, D. Grasso $^{2,3}$, H. Lutjens $^{7}$, P. Maget ${ }^{6}$, S. Nowak ${ }^{1}$, O. Sauter ${ }^{5}$, C. Sozzi ${ }^{1}$ and the EUROfusion MST1 Team *

1 Istituto di Fisica del Plasma - CNR Via R.Cozzi 53, 20125, Milano, Italy

${ }^{2}$ Istituto dei Sistemi Complessi - CNR Via dei Taurini 19, 00185, Roma, Italy

${ }^{3}$ Dipartimento Energia, Politecnico di Torino Corso Duca degli Abruzzi 24, 10129, Torino, Italy

4 Department of Astrophysical Sciences and Princeton Plasma Physics Laboratory,

Princeton University, Princeton, New Jersey 08544, USA

5 Ecole Polytechnique Federale de Lausanne (EPFL),

Swiss Plasma Center (SPC), 1015 Lausanne, Switzerland ${ }^{6}$ CEA,

IRFM, F-13108 Saint Paul-lez-Durance, France.,

7 Centre de Physique Theorique, Ecole Polytechnique, CNRS, France.

\footnotetext{
**See the author list of Overview of progress in European Medium Sized Tokamaks towards an integrated plasma-edge/wall solution by H. Meyer et al., to be published in Nuclear Fusion Special issue: overview and summary reports from the 26th Fusion Energy Conference (Kyoto, Japan, 17-22 October 2016)
} 


\section{ABSTRACT}

The disruptive collapse of the current sustained equilibrium of a tokamak is perhaps the single most serious obstacle on the path toward controlled thermonuclear fusion. The current disruption is generally too fast to be identified early enough and tamed efficiently, and may be associated to a variety of initial perturbing events. However a common feature of all disruptive events is that they proceed through the onset of MHD instabilities, and field reconnection processes developing magnetic islands which eventually destroy the magnetic configuration. Therefore the avoidance and control of magnetic reconnection instabilities is of foremost importance and great attention is focussed on the promising stabilization techniques based on localized rf power absorption and current drive. Here a short review is proposed of key aspects of high power rf control schemes (and specifically Electron Cyclotron Heating and Current Drive ECH/ECCD) for tearing modes, considering also some effects of plasma rotation. From first principles physics considerations, here new conditions are presented and discussed to achieve control of the tearing perturbations by means of high

power $\left(P_{E C} \geq P_{o h m}\right)$, in regimes where strong nonlinear instabilities may be driven, such as secondary island structures, which can blur the detection and limit the control of the instabilities. Here we consider recent work which motivates the search of improvement of some traditional control strategies, namely the feedback schemes based on strict phase tracking of the propagating magnetic islands.

\section{INTRODUCTION}

The tearing modes have been the subject of extensive studies for many years [1-5]. The first basic linear and nonlinear theory has been subsequently extended to neoclassical regimes [6-8], with bootstrap current effects. Recently the physics understanding has been enriched by new findings on nonuniformity effects on finite magnetic islands, of pressure and temperature associated with energy input [ECRH] and loss (e.g. by radiation) [9-15], rotation and [16-21], as well as by findings on small scale topological effects of the reconnection [22, 23]. The mutual interaction of tearing modes with shielding effects and coupling and the symmetry breaking effects on global and local plasma rotation have gained great attention, for their important consequences. One of the most promising methods of controlling magnetic islands 
is based on driving directly into the magnetic island a current, by absorption of rf waves mainly at the electron cyclotron frequency (ECCD) [24, 25], with a $(m, n)$ helical component counteracting the destabilizing current perturbations [26]. Successful ECCD experiments of NTM control, with different methods, have been carried out in ASDEX Upgrade [30-32], JT60U [33], FTU [34], DIII-D [35, 36], KSTAR [37],TCV[38] and EAST [39], and comprehensively reviewed in $[40,41]$ In a tokamak the task of possible prevention, or effective control of low $(m, n)$ order magnetic reconnection instabilities can rely on few knobs which can be associated just with a few state variables, in a coarse grained picture of the processes. Progress in the physics understanding of the local and averaged effects of rf power absorption is needed to identify the most important limits and bounds; then within these limits one can conceive and design (with state of the art engineering) control systems as insensitive to disturbances as possible (robust), but still responding to the physics to be controlled. The realization of a reliable control scheme based on the steered launch and absorption of high rf power in very precise positions in the tokamak plasma, requires numerous diagnostics and control concepts for robust (largely insensitive to external and internal disturbances) real time $(\mathrm{r}-\mathrm{t})$ operation. The technical implementation of such systems leads to very complex architecture,[30, 31, 42-44] and this motivates a careful revisitation of the underlying principles to achieve efficiency and reliability. The actual design is the task of professional control engineers, but the formulation of the problem requires the work of expert physicists, capable of isolating the dominant and subsidiary processes, specifying the relevant parameter space, the state and control variables and the eventual acceptable structure of a simplified plant description.

The basic tasks for the prevention or control of collapsing events, requires successful means of detection of the unstable modes, in spectrum, amplitude, phase and frequency as well as in choice of strategies of constraint. For instance the generic (albeit formidable) goal of stability must be substantiated in defining the actual desired range of variation of the (main) state variable (e.g. magnetic island width), and the restriction due to subsidiary (unwanted) processes. In this respect it should be borne in mind that the application of intense external coercive means (e.g. boundary magnetic perturbations or ECCD) may lead to violation of the constant $\psi$ regime, on which the governing equation for island evolution is based [1-4]; entering a non constant $\psi$ regime implies the possibility of driving instabilities growing faster than the current diffusion process out of the reconnecting region, splitting the fragile 
$\mathrm{X}$-points into two Y-points and forming secondary islands which blur the identification of the phase. This leads to expect a limit on the amplitude and localization of the externally applied perturbation. The occurrence of multiscale effects (in space and time), as discussed in $[22,23]$, on one hand, increases the difficulties of selecting control strategies, while, on the other, it offers several possibilities of diagnosing the unstable state. Furthermore in realistic tokamak regimes, account should be taken of plasma toroidal rotation, intrinsic or driven, which alters the stability picture.In the first section the framework equations are presented, from which specializedand simplified models are deduced,.In the second section the effects of toroidal rotation on magnetic reconnection are briefly diascussed, in relation with new recent results. In the next section the finite island evolution through the Rutherford equation [2] is recalled, with discussion of the physical role and shortcomings of destabilizing and stabilizing terms. The fourth section introduces the novel discussion of the nonlinear effects of intense rf driven current on the process of control of reconnection. The emerging physical limits on mode phase and amplitude control lead to analyse and suggest alternative robust approaches, discussed in the last section.

\section{EQUATIONS FOR RESISTIVE MAGNETIC PERTURBATIONS}

We refer here for completeness to the following rather general MHD equations, applying, for the purpose of this work, suitable symplifications, when necessary:

$$
\begin{gathered}
\varrho\left[\partial_{t} \boldsymbol{v}+\boldsymbol{v} \cdot \boldsymbol{\nabla} \boldsymbol{v}\right]=-\nabla p-\nabla \cdot \boldsymbol{\Pi}+\boldsymbol{J} \times \boldsymbol{B} \\
\partial_{t} \boldsymbol{B}=\boldsymbol{\nabla} \times(\boldsymbol{v} \times \boldsymbol{B})-\boldsymbol{\nabla} \times\left(\eta\left[\boldsymbol{J}-\boldsymbol{J}_{b o o t}-\boldsymbol{J}_{C D}\right]\right) \\
\frac{3}{2} \partial_{t} p+\nabla \cdot\left[\frac{5}{2} p \boldsymbol{v}\right]=-\nabla \cdot \boldsymbol{q}+\boldsymbol{v} \cdot \boldsymbol{\nabla} p-\boldsymbol{\Pi}: \nabla \boldsymbol{v}+Q+P_{r f}
\end{gathered}
$$

where $\boldsymbol{v}$ is the plasma MHD velocity, $p$ the plasma pressure, $\boldsymbol{\Pi}$ the pressure tensor, $\boldsymbol{B}$ the magnetic field and $\varrho$ the mass density which is assumed constant both in space and time. In equation (2) the plasma resitivity $\eta$ may be considered parametricallydependent on temperature, while the current density $\boldsymbol{J}$ is defined by $\mu_{0} \boldsymbol{J}=\boldsymbol{\nabla} \times \boldsymbol{B}$ (hereafter we normalize $\left.\mu_{0}=1\right)$. In equation $(3), \boldsymbol{q}$ is the heat flux, $\mathrm{Q}$ is the energy loss term and $P_{r f}$ represents the rf heating power input. Actually in the following model the effect of the heat balance will just influence some plasma parameters, temperature dependent in quasi-steady state conditions.Also the $\boldsymbol{\Pi}$ the pressure tensor in (2) and (3) is neglected in the following, except 
for its footprint in the bootstrap corrections to the current density represented by $\boldsymbol{J}_{\text {boot }} ; \boldsymbol{J}_{C D}$ indicates the externally driven ECCD current. In addition we consider here also a sheared equilibrium toroidal flow $\boldsymbol{v}_{0}=R \Omega(r) \hat{\boldsymbol{z}}$ ( $R$ is the major radius and $\hat{\boldsymbol{z}}$ is the unit vector along the longitudinal direction), satisfying $\varrho\left[\boldsymbol{v}_{0} \cdot \boldsymbol{\nabla} \boldsymbol{v}_{0}\right]=-\boldsymbol{\nabla} p_{0}+\boldsymbol{J}_{0} \times \boldsymbol{B}_{0}$. For small non axisymmetric magnetic perturbations, it is convenient to use the non orthogonal curvilinear coordinate system $u^{i}=(V(\psi), \theta, \zeta)$, whose arbitrary Jacobian $\sqrt{g}=1 / \nabla V \times \nabla \theta \cdot \nabla \zeta$ here is chosen to be unitary, with $B \cdot \nabla \vartheta=\psi^{\prime} / \sqrt{g}$ where the prime indicates the derivative respect to $\mathrm{V}$. The total magnetic field can be represented in the general form:

$$
\mathbf{B}=\psi_{t}^{\prime} \nabla V \times \nabla \vartheta-\psi^{\prime} \nabla V \times \nabla \zeta=\psi^{\prime} \nabla \times(V \nabla \alpha)(4)
$$

where $\alpha=q \vartheta-\zeta$ is a Clebsch magnetic field line label, $\psi_{t}$ and $\psi$ are the toroidal and poloidal magnetic fluxes, such that the ratio $\psi_{t}{ }^{\prime} / \psi^{\prime}$ is constant at a given flux surface. We introduce the safety factor defined as $q=\psi_{t}{ }^{\prime} / \psi^{\prime}$. It is known that magnetic perturbations described by linearizing the above system, in absence of an equilibrium flow and of an external current source $J_{C D}$, may grow unstable at rational surfaces $q\left(r_{s}\right)=\frac{m}{n}$, developing magnetic islands. The first question addressed here is on the role that a background sheared flow may have on these linear resistive instabilities. From previous studies [16, 19, 20] a variety of results is available for discussion. Here we want to propose a first principles discussion based on particular, albeit artificial, incompressible flow and q profiles, that have the merit of leading to exact solutions. Viscosity effects (arising from $\boldsymbol{\nabla} \cdot \boldsymbol{\Pi}$ ) are dropped and the choice $\nabla \cdot \boldsymbol{v}=0$ is along the line of Ref.[45]. The basic tool is a form of Newcomb's equation obtained in cylindrical approximation from the linearization of eqs.(1)-(3) written in terms of the reduced MHD variable $\psi=-\frac{r B_{0} k_{\|}}{m} X$, modified by the presence of a rotation with a simple family of profiles for $k_{\|}$and rotation. The profiles employed in our analysis are the following

$$
\begin{aligned}
& k_{\|}(r)=\frac{n s}{\lambda}\left[1-\left(\frac{r}{r_{s}}\right)^{\lambda}\right] \\
& \Omega(r)=\Omega_{0}\left[1-\left(\frac{r}{r_{s}}\right)^{\lambda}\right]
\end{aligned}
$$

where $n$ is the toroidal mode number, $r_{s}$ is the resonant point, $s$ denotes the magnetic shear at $r_{s}$ and the parameter $\lambda$ labels the profiles determining their steepness [18]. Note that we chose a reference frame which is moving along the longitudinal direction in a such way that the rotation frequency vanishes at $r_{s}$ (this is allowed within the cylindrical approximation). 
Exact solutions in terms of hypergeometric functions can be found and an analytic expression of the classical instability index $\Delta^{\prime}$, with sheared flow, is obtained:

$$
\begin{aligned}
r_{s} \Delta^{\prime} & =-\left(\frac{m^{2}-\mu^{2}}{r_{s} \lambda}\right) \pi \cot [\pi(m-\mu) / \lambda] \\
\mu & =\left(m^{2}+2 \lambda+\lambda^{2}[1+\Theta(y)]\right)^{1 / 2}
\end{aligned}
$$

where $\Theta(y)=\frac{2 y^{2}}{\lambda^{2}\left(1-y^{2}\right)}$. It is found that the key parameter is the ratio $y=\frac{\Omega^{\prime} / \omega_{A}}{q^{\prime} / q}$ of toroidal rotation shear and magnetic shear $[18,19]$. For $y \ll 1$ a weak destabilizing effect due to rotation shear is present, and generally the small $m$ tearing modes are unstable $\left(\Delta^{\prime}>0\right)$, while large $m$ 's are stable $\left(\Delta^{\prime}<0\right)$. For $y \sim 1$ a window of stability exists for all $m$. The response of the nonlinear growth rate $d \ln (w) / d t$ of the neoclassical tearing modes (NTM) to rotation shear, reflects the classical behaviour in reducing the unstable $w$ range, but rotation alone does not seem to provide a reliable control knob. These exact results [18] are in agreement with the form of $\Delta_{0}^{\prime}$ derived in toroidal geometry using the WKB approximation $(m \gg 1)$, see Ref. [20].

\section{ECH AND ECCD EFFECTS IN THE GENERALIZED RUTHERFORD EQUATION (G.R.E.)}

The main task of a theoretical study of rf control of tearing instabilities, in the observable Rutherford phase, is the estimate of the necessary driven current, e.g. the rf power necessary to reduce the state variable $w(t)$ and to design real-time strategies for the rf launching for an effective power deposition and possible tracking of the moving island. A basic question for the design of a control concept is the order of the fastest and slowest time scales of the processes to be controlled (fast reconnection, slow nonlinear growth and saturation, island rotation period), and the associated space scales suggesting how sharp the focusing, radial and/or angular, should be. Moreover, in addition to all the physical scale lengths mentioned above, one has to consider also that the rf-driven current forces another typical scale length, the ECH absorption depth $w_{c d}$ as defined,for instance, in Ref[15]. This length depends on the wave beam launching and propagation conditions and plasma equilibrium quantities, such as density and temperature. The equation for the evolution of magnetic islands with width $w=4 \sqrt{\psi_{s} L_{s} / B}$, larger than the tearing layer is known as Rutherford equation [2].In this expression $\psi_{s}$ is the reconnected helical flux at the q rational surface and $L_{s}$ the local 
magnetic shear length. The equation for $\mathrm{w}(\mathrm{t})$ is obtained from the rate of flux reconnection with suitable averaging of the Faraday-Ohm equation (2) and it has been generalized to include neoclassical effects, plus the effect of the ECH/ECCD and is coupled to equation for the island rotation frequency $\omega$ in the lab frame, similarly obtained from the equation 1 (see, e.g., $[47,49-52])$. Here it is convenient to present it in a form currently used in modelling $[47,48]$ :

$$
\begin{gathered}
g_{1} \frac{\tau_{R}}{r_{s}} \frac{d w}{d t}=r_{s}\left[\Delta_{0}^{\prime}+\frac{a_{b s} \Delta_{b s_{0}} w}{w^{2}+w_{d}^{2}}-\frac{a_{G G J} \Delta_{G G J_{0}}}{\sqrt{w^{2}+0.2 w_{d}^{2}}}+\frac{a_{p o l} \Delta_{p o l_{0}} \rho_{\theta i}^{2} w}{w^{4}+w_{\rho}^{4}} \frac{\bar{\omega}\left(\bar{\omega}-\omega_{* i}\right)}{\omega_{* e}^{2}}-\Delta_{r f}^{\prime}-\Delta_{w}^{\prime}\right] \\
I_{\phi} \frac{d \omega}{d t}=-T_{0 e m}\left(\frac{w}{r_{s}}\right)^{4} \frac{\left(\omega \tau_{w}\right)}{1+\left(\omega \tau_{w}\right)^{2}}-\left[\omega-\omega_{T}\right] \frac{d I_{\phi}}{d t}-4 \pi^{2} R^{3} r_{s}^{3} \varrho \nu \frac{w}{w^{2}+w_{\nu}^{2}}\left[\omega-\omega_{T}\right]+T_{e x t} \\
\frac{d \varphi}{d t}=\omega
\end{gathered}
$$

Here $\tau_{R}, \tau_{w}$ are the resistive dffusion time scale and the wall constant [48], $\nu$ is a viscosity associated with momentum diffusion and $\bar{\omega}=\omega-\omega_{E}$, where $\omega_{E}, \omega_{* e, i}$ are the electric EXB drift and diamamagnetic frequencies and $\omega_{T}=\omega_{E}+\omega_{* i}+\kappa\left(c k_{\theta} T_{i}^{\prime} / e B_{0}\right)$, with $\kappa$ a neoclassical coefficient $\mathrm{O}(1)$ [47]. In the torque balance equation, $I_{\phi}=4 \pi^{2} \varrho R^{3} r_{s} w$ is the moment of inertia of the rotating island and the constant $\mathrm{T}_{0 e m}=4 \pi^{2}\left(R / \mu_{0} r_{s}\right)\left[B r_{s} / 16 R q L_{q}\right]^{2} k_{\theta}\left(r_{s} / d_{w}\right)^{2 m}$ is the amplitude of the electromagnetic (em) torque due to eddy currents in the wall,assumed with circular cross section with minor radius $d_{w}$ and $k_{\theta}=m / r_{s}$ is the mode wavenumber. The last term $T_{\text {ext }}$ in eq.(10) is introduced here as a hypothetical external torque that could be applied to control the mode rotation [53]. The term $\Delta_{0}^{\prime}$ represents the amplitude of the jump of logaritmic derivative of $\psi$ across the $q=m / n$ surface [1-4] and in presence of a background toroidal rotation must embody the physics described in Eq.7. The dimensionless terms $\Delta_{b s_{0}}=\beta_{p} \sqrt{\epsilon}\left|L_{q} / L_{p}\right|, \Delta_{G G J_{0}}=\beta_{p} \epsilon^{2} L_{q}^{2} /\left(r_{s}\left|L_{p}\right|\right), \Delta_{p o l_{0}}=\beta_{p}\left(L_{q} / L_{p}\right)^{2} g\left(\epsilon, \nu_{i i}\right)$, represent, respectively, the bootstrap current $J_{b s}$ effect, [6], a toroidicity effect [3], and the third term represents the polarization current due to an effect of ion inertia [8,9], which is important at the onset of the NTMs. In the other denominators $w_{\nu} \sim w_{\rho}=O\left(\rho_{\theta i}\right)$ represent a physical lower limit island width, $L_{q}, L_{p}$,represent the scale lengths of the q and pressure profiles, $\epsilon=r / R$ is the inverse toroidal aspect ratio, $\beta_{p}=\mu_{0} p / B_{p}^{2}$ and $g\left(\epsilon, \nu_{i i}\right)$ is a function of collisionality specified in $[8,9]$. A conventional, accepted evaluation [50] of the other coefficients is $a_{b s}=2.6, a_{G G J}=6$. The last term $\Delta_{w}^{\prime}=2 k_{\theta}\left(r_{s} / d_{w}\right)^{2 m} \frac{\left(\omega \tau_{w}\right)^{2}}{1+\left(\omega \tau_{w}\right)^{2}}$, with label w for 
wall, gives a small stabilization due to the induced currents in the first wall [48]. The quantity $w_{d}$ in the second (bootstrap current) and third [3] terms of the first equation represents a lower limit of the island width related to the finite ratio of heat conductivities field along and across the B field $\left(\chi_{\|} / \chi_{\perp}\right)$, and governs the incomplete flattening of the temperature profile within the island separatrix $[55,56]$. It is often replaced by the value $w_{\text {marg }}$ below which NTMs self extinguish. The portrait of the stability conditions in the neoclassical collisional regimes shown in the phase plane $(\mathrm{dw} / \mathrm{dt}, \mathrm{w})$ of Fig.1(top), where the meaning of the nomenclature $w_{t h}, w_{\text {marg }}, w_{\text {sat }}$ and the interval where $d w / d t>0$ is apparent. A neoclassical tearing mode, at low $\beta_{p}$ is linearly and nonlinearly stable, with $\Delta_{0}^{\prime} \leqslant 0$. At higher $\beta_{p}$ the neoclassical NTMs are metastable, without an island, until a seed perturbation (presumably of the same helicity) triggers the growth, proportional to $\beta_{p}[6,46,57]$. In Fig.1(bottom), the effect is shown of toroidal sheared rotation on the stability domain, discussed in Section III [18].Recent results show that in condition of low magnetic shear in the plasma core, finite pressure gradient effects can excite infernal modes which can trigger tearing sidebands [46] through toroidal coupling. The rf power term physically consists of contributions describing the helical and axisymmetric current drive, and of localized heating is: $\Delta_{r f}^{\prime}=\Delta_{C D}^{\prime}+\Delta_{E C H}^{\prime}$. According to [12] the calculation for the helical contributions should be given by $\Delta_{C D}^{\prime}=\frac{16 L_{q}}{\pi B_{p} w^{2}} \int_{-w / 2}^{w / 2} d x \int_{-\pi}^{\pi} d \xi J_{m, n C D} \cos (\xi)$, with $\xi=m \theta-n \phi$ with integration restricted to the island region, while the contribution of the axisymmetric current results from integration outside the same region. Since the integrands of both terms are nearly identical, the usual expression for $\Delta_{C D}^{\prime}$ obtained by integrating from $x=-\infty$ to $x=+\infty$ contains the effect of both the helical and axisymmetric EC driven current. The contribution of the rf drivenl current is conveniently written in terms of $I_{C D}, I_{p}$, the total $\mathrm{rf}$ and plasma currents, as $\Delta_{C D}^{\prime}=32 \frac{I_{C D}}{I_{p}} \frac{L_{q}}{w_{c d}^{2}} \eta_{m n}\left(\frac{w}{w_{c d}}\right) G_{C D}\left(r_{d e p}, \frac{w}{w_{c d}}\right)[14,15]$. The ECCD efficiency $\eta_{m n}$ appearing in this term $([14,49,52])$ is best fitted analytically by $\eta_{m n, C W}\left(w / w_{c d}\right)=\frac{0.25}{1+(2 / 3)\left(w / w_{c d}\right)}[14]$ for the constant $(\mathrm{CW})$ rf application and by $\eta_{m n, 50 \%}\left(w / w_{c d}\right)=0.45 \tanh \left[0.4\left(w / w_{c d}\right)\right]\left(\frac{w_{c d}}{w}\right)^{2}$ for the phased modulation [14]. The function $G_{C D}\left(r_{d e p}, \frac{w}{w_{c d}}\right)$ accounts for the radial misalignment effects[13]. In addition, the local heating effect of the EC waves power absorbed (by the electrons), gives $\Delta_{E C H}^{\prime} \propto \frac{16 L_{q} \sqrt{\pi}}{w_{c d}^{2} B_{p}} P_{E C} \eta_{H}\left(\frac{w}{w_{c d}}\right)$, due to modification of $J_{e q}$ through the resistivity. It should be noticed that an excess of the axisymmetric component of $J_{C D}$ may shift the minor radius location of the $q=m / n$ surface and consequently also the island "O" point, resulting in a reduced efficiency . Of course when using the expressions for CW 
ECCD, the island phase tracking is irrelevant, but the model becomes invalid when the mode locks. From the steady state of eq.9 it appears that for locally peaked temperature profiles, the heating helps reducing the saturation width of the island even if it does not suppress it [49]. For ITER-like plasma parameters $\left(R_{0}=6.3 m, a=2 m, B_{0}=5.3 T, r_{s} \sim\right.$ $1.6 m, I_{p}\left(r_{s}\right)=11 M A, T_{e}\left(r_{s}\right)=7 k e V, n_{e}\left(r_{s}\right) \sim n_{i}\left(r_{s}\right)=9.5 \cdot 10^{19} m^{-3}, \tau_{R}=284 s, \beta_{p o l}=$ $\left.0.7, w_{\text {sat }}=0.21 \mathrm{~m}, w_{d}=w_{\text {marg }}=0.03 \mathrm{~m}, J_{C D}=0.015 \mathrm{MA} / \mathrm{m}^{2}, w_{c d}=0.04 \mathrm{~m}\right)$, in Fig.2 it is shown that the contribution of the axisymmetric driven current and of the heating part are of the same order of that of the helical current, in balancing the destabilizing bootstrap $\Delta_{b s 0}^{\prime}[12]$. Since these effects are independent of the island phase stringent requirements on phase tracking appear less motivated. Furthermore, finite magnetic islands are actually asymmetric with respect to the rational q surface, and the asymmetry is equivalent to a current perturbation which can either have stabilizing effects [10, 11] or destabilizing, when associated with thermal losses.A current perturbation due to variations of the local (Spitzer) resistivity, consequent to radiative cooling of the island interior, has been shown to be destabilizing [11] in combination with asymmetry. Replacing the radiative energy losses by EC heating within a band encompassing the reconnection layer seems therefore a reasonable way to counteract these instabilitiies, also by freezing the reconnection process and it combines favorably with the effect of axisymmetric $J_{C D}[11,12]$, both being phase independent. For a realistic ITER-like scenario,the full curves in the plot (dw/dt,w) of Fig.3 show the full effect of ECCD [12] for different values of injected $J_{C D}$ and Fig.4 shows the modest difference between the case of a CW application and that of a $50 \%$ modulation, perfectly phased. In the frame of the G.R.E, one key question is whether to apply a prompt intervention to suppress the island of width $\mathrm{w}$ as soon as the instability is detected, or apply continuous pre-emptying control of a finite island within chosen bounds [12, 25, 26, 30, 32, 52, 54, 57]. Conventionally an estimate of the power $P_{C D}$ required to quench the island growth is obtained by setting to zero the r.h.s of eq.9, assuming that the power deposition is well aligned with the mode resonant surface, while in case of modulated ECCD in addition the high power period is assumed to be accurately synchronized with the passage of the island O-point through power deposition region. This can possibly obtained by entraining the modes by external rotating fields, as done in some exploratory experiments $[36,53]$. There the interesting technique has been demonstrated, of shifting the phase of a locked island by external magnetic fields to allow the shining of CW EC wave beams on the O-point of the locked mode greatly im- 
proving the efficiency over any value achievable for a rotating mode. However this approach is hardly applicable to a reactor grade device, for prompt and robust actiion. The required power for island suppression is given by the expression:

$$
P_{E C \min }=\max _{(w)}\left(\frac{w_{\text {sat }} w}{w^{2}+w_{\text {marg }}^{2}}-1\right) \frac{a_{b s}}{a_{C D}} \frac{4(1-f) w_{c d}}{\sqrt{\pi} w_{\text {sat }}} \frac{1}{\eta_{C D} \eta_{N T M}}
$$

where $\eta_{N T M}$ is the estimated ratio (Ref.[25-27]) $J_{C D} / J_{b s}$ of the local ECCD current driven by $1 \mathrm{MW}$ of power to the bootstrap current density and $f=a_{G G J} \Delta_{G G J 0} / a_{b s} \Delta_{b s 0}<1$, neglecting the $\Delta_{p o l}$ and $\Delta_{w}^{\prime}$ contributions. Many discussions have been made on the advantages of modulating the rf power to deposit the $J_{C D}$ as close as possible to the O-point in synchronism with the island rotation. Actually the parallel transport is virtually instantaneous, such that the driven current density becomes a flux function, on the island flux tubes intercepted anywhere by the rf beam having a deposition spot of finite angular and radial width, and an automatic modulation occurs, encompassing the O-point for a deposition in the range $0<\alpha<\pi$, if the radial deposition is within $w_{c d} \sim w / 2$. So the ECCD efficiency varies moderately between a CW and a phase modulated case [28] and what really matters is minimizing the radial mismatch within a range of the order proposed for instance, in Ref.[25, 26]. In the ITER-like case presented, the control of a 2/1 NTM using EC power modulation is obtained with a reduction of power of less than $\sim 10 \%$ (about $400 \mathrm{KW}$ ), because $w \geq w_{c d}$. The ECCD efficiency is illustrated in Fig. 5 , as function of the ratio $w / w_{c d}$. The adverse effect of a radial misalignment is visible in Fig.5: with deposition error $\delta R \sim 0.016 m$ from the island O-point, the efficiency is much reduced in the interval $w \leq w_{c d}=0.04 m$. It recovers the trend of Fig.5(top) for $w>w_{c d}$ but then it is too low. To illustrate the destabilizing effect of a radial misalignment, which favors the rf power absorbtion across the separatrix, Fevrier et al [29] have proposed a heuristic correction to the efficiency $\eta_{C D} \propto\left(1-\left(\frac{\delta R}{\alpha}\right)^{2}\right) \exp \left[-\left(\frac{\delta R}{\beta}\right)^{2}\right]$, where $\alpha, \beta$ are profile scale lengths. In conclusion, when the measurements of amplitude $\left(\propto w^{2}\right)$, and phase are available with sufficient accuracy, the G.R.E provides a very useful model of the process to be controlled, adopting relatively well established systems $[31,36$, $42,43]$. However for the reliability of the control system, with high power circulation, also consideration is needed of possible nonlinear parasitic processes, discussed in the next section 


\section{ECCD MAGNETIC ISLAND SUPRESSION AS CONVERSE OF A NONLINEAR FORCED RECONNECTION PROBLEM}

In this section the attention is addressed to subtler physical effects which may occur on smaller space scales, albeit in a restricted range of operative parameters. We address numerically the problem of the fully nonlinear evolution of a magnetic island, subject to the control of deposited rf helical current, establishing an analogy with a converse of the well known Hahm,Kulsrud,Taylor ( HKT) problem of forced reconnection where the forcing boundary conditions of Ref.[58, 59] are replaced by the effect of a suitable $J_{C D}$, and the intitial state is replaced with one with finite a magnetic island. It is convenient to isolate the problem in the framework of the simplified Reduced Resistive MHD model in 2D slab geometry,described in ref.[23],deducble from Eqs. $(2,1)$ by standard procedures. The magnetic field is expressed through a flux function $\psi$ and the velocity field through a stream function $\phi$ (electrostatic potential). Dimensionless variables are defined and subsequently used dropping hats: $\hat{x}=x / a, \hat{t}=\tau / \tau_{A}, \hat{\psi}=\psi / a B_{0}, \hat{\phi}=\phi / a^{2} \tau_{A}, \hat{J}_{C D}=J_{C D} a / B_{0}, S=\tau_{R} / \tau_{A}$. In this dimensionless model $\eta=S^{-1}$, the inverse Lundquist number, and $\nu=P \eta$, with $\mathrm{P}$ the Prandtl number. The controlling rf current distribution on the intercepted flux surfaces, namely ECCD, [24], can be modeled as a function of $\psi$, which, without loss of generality can be chosen to be of the form:

$$
J_{C D}=J_{0} \exp \left[-\frac{\left[\psi(x, y, t)-\psi_{O}(t)\right]^{2}}{\psi_{c d}^{2}}\right]
$$

where $\psi_{O}$ is the flux value at the island "O" (elliptic ) point and $\psi_{c d}$ is the rf current channel size in flux coordinates. It should be observed that when the island, in the constant $\psi$ regime, shrinks below some critical value,related to the conditions found in [22], if the driven current has a scale size (absorption depth) comparable with this critical value, it can drive the perturbation into a non-constant $\psi$ regime, where marginal, nonlinear instability conditions can be reached for tearing unstable current sheets and secondary island structures [22]. Illuminating results have been obtained by carrying on a set of numerical experiments on the response to rf driven current,of magnetic island arising from a spontaneous reconnection event in a static, linearly unstable Harry's pinch equilibrium $\phi_{e q}=0$, $\psi_{e q}(x)=-\log (\cosh (x))$. The current drive is applied continuously starting from a large nonlinear magnetic island. Fig.6 shows the magnetic configuration at the time when the ECCD injection starts (left frame) and the time evolution of the magnetic island area in 
absence of control.

Different widths of the ECCD beam deposition have been adopted, while the initially injected total ECCD current, $\int J_{C D}\left(x, y, t_{1}\right) d x d y$, where $t_{1}$ is the initial deposition time, is the same. The center of the ECCD beam is constant and located at the O-point of the magnetic island at $t=t_{1}$. Fig.7 shows, from top to bottom, the effect of the ECCD beam injection for three different values of $\hat{w}_{c d}=b \cdot \hat{w}\left(t_{1}\right)^{2} / 2$, with $b=0.5,1$, 2, where $\hat{w}\left(t_{1}\right)$ is the (dimensionless) magnetic island half width at $t=t_{1}$. In each row, corresponding to a specific value of $b$, the left frame shows the plasma current at a fixed time with, superimposed, the magnetic surfaces crossing at the X-points. The right frame shows the evolution of the reconnected area, i.e. the area of the region enclosing magnetic surfaces with a different topology compared to the equilibrium configuration. It reduces to the area of a magnetic island when a single mode dominates over the others.

We observe that in all the cases considered here the system moves towards a stationary configuration where the area of the reconnected region is comparable with the area of the magnetic island at the initial deposition time. However the current control has a significant effect on the change of the magnetic topology compared with the initial magnetic island. Moreover this change appear to be strongly dependent on the value of the beam width. The numerical analysis shows that the new topology is the results of a complex dynamics induced by the continuous deposition of the $J_{C D}$. After an initial phase when the $J_{C D}$ reduces effectively the magnetic island, in fact, the small scale current layers induced by the external control current along the null axis $x=0$ give rise to plasmoid like secondary structures. These structures grow and recombine on fast time scales, leading to a continuous change of the magnetic topology untill the saturation is reached. Note that the smaller the $b$ parameter, the more lively the dynamics. Therefore the striking result is that the ECCD current injection, meant to suppress the Rutherford magnetic islands, can lead to formation of a secondary island chain on the scale of $w_{c d}$, as shown in Fig.7. In practice, for ITER-like cases similar to the example presented, the expected beam focussing $\hat{w}_{c d} / \hat{w}\left(t_{1}\right) \sim w_{c d} / w_{\text {marg }} \geq 1$ is broad and should avoid this secondary forced reconnection. Nonlinear formation of secondary islands, expected in systems with large amount of free energy, has not been often documented in tokamak experiments. However interesting observations have been recently reported in JET (without ECH) and COMPASS [60] and in FTU [61] in presence of ECH. In designing control systems based on delivering large rf power with sharply focussed beams, 
the associated nonlinearities should be considered. The possible occurrence of such nonlinear substructures, shown in Figs.7, clearly hampers the use of the phase as a measurable and controllable variable.

\section{DETECTION AND CONTROL ISSUES: FEEDBACK AND OPTIMAL CONTROL POLICY}

A vast literature and different approaches exist on the diagnostics of the magnetic instabilities, addressing the question of identifying the fundamental state variables: frequency (and phase), $\mathrm{m} / \mathrm{n}$ helicity and amplitude of the modes, as well as their "radial" location. Here we refer to recent works offering a panoramic view of the various issues [31, 42-44, 53, 63]. In the previous sections it has been shown that full suppression of NTMs requires essentially a good accuracy of the EC power deposition position at the $q=m / n$ surface . The detection of the island location in contemporary tokamaks is a complex procedure based on measuring the temperature fluctuations associated with the magnetic islands using Electron Cyclotron Emission (ECE) radiometry, associated with preliminary equilibrium reconstruction and use of correlation methods, with the Mirnov coils signals, analysed in r-t by a digital PLL (phaselocked loop) for frequency identification and Singular Value Decomposition (SVD) methods for mode helicity identification [34, 43, 61-63]. The diagnostic procedure includes specifically by: r-t monitoring of beams launching angles ( with nuclear resistant gauges); measurement of deposition radii $r_{i-\text { dep,meas }}$ (e.g. by the response to probing modulated ECH),followed by estimate of the i-th $r_{i-d e p, e s t}$ (by ray tracing [64]), and assimilation (by Bayesian filtering $[31,34])$ of $r_{i-d e p, m e a s}$ and $r_{i-\text { dep,est }}$ to obtain a final $r_{i-\text { dep,observ }}$ with properties of continuity and reliability of the observation to be fed to the control loop. The required $E C R H / E C C D$ deposition accuracy must be reached within a fraction of the maximum allowed "latency", defined as the time difference between island seeding of the NTM and the start of mode suppression using ECCD, that still results in full suppression of the mode,[43, 44].This is clearly a machine dependent experimental characteristic difficult to asses a priori and source of uncertainty. A low latency detection method of the mode position has been propposed based on receiving the ECE radiation via the equatorial port plug and along the line-of-sight of the ECCD launcher[44].A very interesting variant has been applied on D-IIID [53] using "oblique ECE", along same direction as ECCD;two ECE channels close to island are suffi- 
cient to track its rotation and radially localize it, and the oblique ECE signal can be used as a waveform generator for modulating the ECCD,in syncronism with the island rotation and in phase with the island O-point.Although encouraging, the applicability of these techniques needs still to be valdated for a tokamak-reactor environment, considering the neutron protection requirements and the reliability, especially for "early detection and prompt action". The variety of the signals to be acquired and processed for the control action, and their intrinsic uncertainties as well as those of the model predictions could be too high for the feedback specification, while the sensitivity is in principle infinite. The direct measurements are characterized instead by a lower uncertainty but with finite sensitivity due mainly to noise. Therefore it has been proposed [61] to use a combination of both using a probabilistic approach based on the Bayesian assimilation in real time of all the information available. An important advantage of using more than one source of information is the possibility to increase the robustness of the estimate by comparing the consistency among the available data. Although modern signal processing techniques can provide excellent tools for frequency identification and phase locking, the possible nonlinear effect, on finite islands, of high localized power, which destroys the mode phase as a useful state variable is a serious problem that compromises robust performance. Another difficult case occurs when the control action is wanted in the early unstable growth interval identified inthe NTM plot ( $d w / d t$ vs. $w$ ) before the maximum (i.e. for $w<w_{\text {marg }}$ ) (see Figs.(3)). Here a very effective mode tracking and amplitude control is most difficult and should be readily applied. The control problem approach presented in this section is fully motivated by these considerations which show the fragilty of the concept of island phase, making hazardous any feedback scheme, based on its r-t tracking as itis too sensitive to external disturbances and to internal nonlinear processes such as those just discussed. An optimal control policy [65] suitably formulated can overcome this difficulty providing robust control with an asynchronous, and (rather) coarse grained action. Here an example is proposed in a general form,for completeness of argument, but simple enough to provide an analytic insight and suggestions for in depth developments. In this interval the NTM control problem can be cast into a linearized form belonging to a general class known in the theory of multistage decision processes [65]. The governing equation (9) for the (dimensionless) $(2 \times 1)$ state vector $\boldsymbol{X}(\boldsymbol{t})=\left[w / r_{s},\left(\omega-\omega_{T}\right) / \omega_{\star}\right]$,(see eq.9) with the initial condition $\boldsymbol{X}(\mathbf{0})=\boldsymbol{X}_{\mathbf{0}}$, and a control vector function $\boldsymbol{U}(\boldsymbol{t})$ after linearization 
can be written as:

$$
\frac{d \boldsymbol{X}}{d t}=\boldsymbol{A} \cdot \boldsymbol{X}+\boldsymbol{B} \cdot \boldsymbol{U}
$$

where the $(2 \times 2)$ matrix $\boldsymbol{A}$ is obtained from the linearized G.R.E. eqs. $(9,10)$, and $\boldsymbol{B}=\left[b_{i j}\right]$ is the $(2 \times 2)$ matrix of control coefficients

$$
\boldsymbol{A}=\left(\begin{array}{cc}
a_{11}>0 & a_{12}<0 \\
a_{21}<0 & a_{22}<0
\end{array}\right)=\left(\begin{array}{cc}
O\left(X_{t}^{-1}\right) & O\left(X_{t}^{-3}\right) \\
O\left(X_{t}^{2}\right) & O\left(X_{t}^{-2}\right)
\end{array}\right)
$$

where $X_{t}=w_{t} / r_{s} \ll 1$ is the NTM (normalized) threshold island value, above which the mode grows unstable [6]. With the dimensionless notation $X_{d}=w_{\text {marg }} / r_{s}, X_{\star}=\omega_{T} / \omega_{\star}$ the coefficients $a_{i j}$, are obtained linearing the equation (9) around typical values slightly above threshold: $a_{11} \sim \frac{\Delta_{b s_{0}}}{X_{d}^{2}}\left[1-3 \frac{X_{t}^{2}}{X_{d}^{2}}\right], a_{12} \sim-\Delta_{p o l} \frac{X_{\star}^{2}}{X_{t}^{3}}, a_{21} \propto \frac{\hat{\tau}_{w} X_{\star} X_{t}^{2}}{1+X_{\star}^{2} \hat{\tau}_{w}^{2}}, a_{22} \propto \frac{\tau_{R}}{\tau_{\mu} X_{t}^{2}}$. The control is switched on with initial state conditions $\mathrm{X}_{01}=X_{1}\left(t=1 / a_{11}\right)>X_{t}, X_{02}<<1$.

The formal problem addressed here consists in reducing the state $\boldsymbol{X}(\boldsymbol{t})$ to zero in a given time $\mathrm{T}$ by a suitable choice of the control $(2 \times 1)$ control vector $\boldsymbol{U}(\boldsymbol{t})$. The latter, when the actuator is the ECH/ECCD launching system, in full generality can be represented in terms of a function of the (normalized) radial misalignment $\delta R / \beta$ (see section IV) between the wave beam deposition position (minor radius) $r_{d e p}$ and the rational q surface $r_{m, n}$ where magnetic islands appear, and of the phase mismatch $\delta \phi$ :

$$
\boldsymbol{U}(\boldsymbol{t})=\left[h \exp \left(-\left(\left[r_{d e p}-r_{m, n}\right]^{2}\right) / \beta^{2}\right), \delta \phi\right]
$$

Near the threshold value $X_{t} \ll 1$ the rank of the system matrix $\boldsymbol{A}$, is full, but the Kalman $(2 \times 4)$ controllability matrix $\boldsymbol{Q}=[\boldsymbol{B}, \boldsymbol{A} \cdot \boldsymbol{B}][66]$ is of full rank (2) only if the coefficients $a_{21}$ proportional to first wall resistivity and $a_{22}$, to perpendicular viscosity , are not vanishing and both columns of the matrix $\boldsymbol{B}$ are non-null. In the example considerd here with just an ECCD actuator,the only non null control term is $b_{11} \propto-\frac{1}{X_{t}^{2}}$; this actually means that the mode rotation and phase is not controllable, and therefore a feedback design should aim primarily at determining the first component of the control vector, $u=U_{1}$ which depends on the radial mismatch $\delta R^{2}=\left[r_{d e p}-r_{m, n}\right]^{2}$, mimicking the EC power absorption line. It is then instructive to explore an approach of optimal control, complementary to the usual feedback schemes, and based,for instance, on the constrained minimization of a suitable "cost function". There are obviously unlimited choices for the "cost function to achieve robust perfomance, but common sense and practicality lead to select the simplest possible. 
To provide an example dealing with the actual state and control variables, that can be easily analyzed, the functional $\mathrm{J}$ has been selected to represent the quenching of an island in a given time, with minimal control strength. This contrasts with the use of maximum power to quench the island, which leads to the phase-flip or secondary islets formation [23, 69], just discussed.

$$
J=\int_{0}^{T} d t\left[u^{2}+1\right]
$$

subject to the fulfilling of the state eq.(14). The optimal control approach, giving the same weight to the control amplitude and time of quench,can combine requirements of robustness and response to the physics. The time $\mathrm{T}$ is a free choice, which reasonably should be of the order of a few growth times of the observed rms (root mean squared) amplitude of the instability. The constrained problem is solved introducing the Hamiltonian from which the adjoint problem is formulated

$$
\begin{aligned}
& H(\boldsymbol{X}, \boldsymbol{p})=1+u^{2}+p_{1} \frac{d X_{1}}{d t}+p_{2} \frac{d X_{2}}{d t} \\
& \frac{d p_{1}}{d t}=-\frac{\partial H}{\partial X_{1}}, \frac{d p_{2}}{d t}=-\frac{\partial H}{\partial X_{2}}, \frac{\partial H}{\partial u}=0
\end{aligned}
$$

For this simple example the instructive solution of the state and adjoint equations is analytic:

$$
\begin{gathered}
X_{1}=X_{01} e^{a_{11} t}+\frac{X_{02} a_{12}}{a_{22}-a_{11}}\left[e^{a_{22} t}-e^{a_{11} t}\right]-u(t) \frac{e^{a_{11} t}}{a_{11}} \sinh \left(a_{11} t\right) \\
X_{2}=X_{02} e^{a_{22} t} \\
u(t)=\frac{a_{11} e^{-a_{11} t}}{\sinh \left(a_{11} T\right)}\left[X_{01} e^{-a_{11} T}+\frac{X_{02} a_{12}}{a_{22}-a_{11}}\left[e^{a_{22} T}-e^{a_{11} T}\right]\right]
\end{gathered}
$$

The parameters of the exercise are deduced from a real TCV discharge [70]. The perturbation of the frequency decays on the timescale $1 / a_{22}$. The Fig.8 (top) shows the solution of eq.(14) during the uncontrolled time interval matched with the controlled one at $t_{0} \geq 1 / a_{11}$. The result shows that a suitable control of the beam can quench the mode amplitude in the case of fixed radial misalignment $\delta R^{2}=$ const.. The general procedure used here shows that, in this case the, uncontrolled, evolution of the frequency, Eq.21, does not hinder the amplitude quenching effect. Eq.22 shows that the control amplitude is proportional to the initial rate of growth of the magnetic island, with a correction due to the 
intial mode perturbed rotation, which here is decaying; since the latter is not precisely measurable, the control geared on the measurement of the mode r.m.s growth rate is sufficient to bring the amplitute to target. A feedback system based on phase tracking may not have suffcient accuracy in the early mode growth stage, within the latency interval, and when secondary structures appear, blurring the phase detection as shown in Fig.7 [61]. Then it is advantageous to complement such systems with optimal control policies. Since it turns out that what is important is the radial focussing, it is interesting to explore a piecewise optimal policy where the control function $u(t)$ is extended allowing for a time dependence of $\left.|\delta R(t)|=\delta_{\epsilon} \mid \Sigma\left(2 \frac{2 t}{\tau_{\Sigma}}-1\right)\right) \mid \sim w$ with $\Sigma(t)$ a triangle waveform representing an intermittent scanning of the neighborhood of the rational surface:

$$
u(t)=\frac{a_{11} e^{-a_{11} t} f_{\Sigma}(t)}{\sinh \left(a_{11} T\right)}\left[X_{01} e^{-a_{11} T}+\frac{X_{02} a_{12}}{a_{22}-a_{11}}\left[e^{a_{22} T}-e^{a_{11} T}\right]\right]
$$

The result in Fig.8 (bottom) shows that a suitable intermittent steering of the beam across the rational surface, pre-determinined by equilibrium identification, with $f_{\Sigma}(t)=$ $\exp \left(-(\delta R(t) / \beta)^{2}\right)$ can substantially quench the mode amplitude also in the extreme case of missing or ineffective control of the phase, reaching robust performance. The implementation in an actual control system, does not require the knowledge of the matrix elements of $\boldsymbol{A}$, but just the r-t measurement of the growth rate of the rms mode amplitude, e.g. from Mirnov coils, possibly correlated with ECE radiometry. In other words, anchoring a suitable feedback action to the minimization of a meaningful cost function rather than relying on ever more complicated diagnostics, can provide robust performance with respect to phase uncertainties. The procedure is expected to be effective also in more complex cases, with disturbances due, for instance,to mode coupling. The results of modelling with the extended MHD code XTOR [29], reproduced in Fig.9 are significantly similar to those of the above model.In both cases (Fig.8, Fig.9) the EC power was not modulated and the oscillations in the response (decaying mode rms amplitide) are just due to the beam sweeping close to the rational q surface. In Refs. $[38,67,68]$ an interesting NTM preemption technique has been demonstrated by applying pulsed power on the mode rational surface of the NTM at the time of the seed-island generating sawtooth crash. preventing the formation of NTMs and suppressing them if they appear. An example of robust performance in recent successful TCV experiments is shown in Fig.10 which fits the picture of the above conceptual model. A practical steering of the rf beam across the target surface could probably be performed 
more easily developing a beam switch akin to the FADIS system [71] which as beeen used succesfully on ASDEX-U to switch the EC power transmission between the transmission lines connected to the upper and lower launchers, respectively, in synhronism with the mode rotation, so that the EC power could effectively be absorbed continuously close the O-point [72]. Another example of NTM amplitude control using a similar method is shown in Fig.11[61]. Here NTMs are reduced in amplitude with an EC beam scanning the $q=m / n$ surface from the low field side.

\section{CONCLUSIONS}

The growing complexity of the achitecture of detection and control systems for tearing instabilities in tokamaks requires a careful selection of priorities in the objectives and tasks. With actuators delivering large, localized power to the system, feedback techniques may be limited by the possible onset of smaller scale phenomena in a non-constant $\psi$ regime, which may blur detection and hinder the stabilization process. The combination of feedback with optimal control policies, can help obtaining the necessary robust performance,safely insensitive to internal and external disturbances.

Acknowledgments. This work has been carried out at the Istituto di Fisica del Plasma CNR, within the framework of the EUROfusion Consortium and has received funding from the Euratom research and training programme 2014-2018 under grant agreement No 633053. The views and opinions expressed herein do not necessarily reflect those of the European Commission.

\section{REFERENCES}

[1] Furth H P, Killeen J and Rosenbluth M N 1963 Phys.Fluids 6459

[2] Rutherford P H 1973 Phys. Fluids 161903

[3] Glasser A H, Greene J M and Johnson J L 1975 Phys.Fluids 1887588 
[4] Ara G, Basu B,Coppi B, Laval G, Rosenbluth M N, and Waddel B V. Annals of Physics 1978 112443

[5] Hastie R J, Sykes A, Turner M and Wesson J A 1977 Nucl.Fusion 17515

[6] Callen J D et al, Proceedings of IAEA Conference Plasma Physics and Controlled Nuclear Fusion Research1987, Vol 2 (Vienna: IAEA) p.157 ).

[7] Fitzpatrick R 1988 Phys. Plasmas 53325

[8] Mikhailovskii A B 2003 Contrib. Plasma Phys. 43125

[9] Smolyakov A I and Lazzaro E 2004 Phys.Plasmas 114354

[10] Smolyakov A I et al 2013 Phys.Plasmas 20062506

[11] White R et al 2015 Phys. Plasmas 22022514

[12] Westerhof E et al 2016 Nucl. Fusion 56036016

[13] De Lazzari D and Westerhof E 2009 Nucl. Fusion 49075002

[14] Sauter O 2004 Phys. Plasmas 11 4808, 2004.

[15] Bertelli N, De Lazzari D and Westerhof E 2011 Nucl. Fusion 51103007

[16] Chen X L and Morrison J P 1990 Phys. Fluids B 2495

[17] Fitzpatrick R 1993 Nucl. Fusion 331049

[18] Brunetti D, Lazzaro E, Nowak S 2017 Plasma Physics and Controlled Fusion 59055012

[19] Chandra D,Sen A,Kaw P et al 2005 Nucl. Fusion 45524530

[20] Sen A et al, 2013 Nucl. Fusion 53053006

[21] Mikhailovskii A B, Pustovitov V D and Smolyakov, A I 2000 Plasma Physics and Controlled Fusion42 309

[22] Comisso L, Grasso D, and Waelbroeck F L 2015 Phys. Plasmas 22042109

[23] Borgogno D, Comisso L, Lazzaro E, and Grasso D 2014 Phys. Plasmas 21060704

[24] Fisch N J and Boozer A H 1980 Phys. Rev. Lett. 45720

[25] Perkins F W, Harvey R W, Makowski M and Rosenbluth M N, "Prospects for electron cyclotron current drive stabilization of neoclassical tearing modes in ITER," 17th IEEE/NPSS Symposium Fusion Engineering (Cat. No.97CH36131), San Diego, CA, 1997, pp. 749-751 vol.2.

[26] Pletzer A and Perkins F W 1999 Phys. Plasmas 61589

[27] Sauter O, Henderson T, Ramponi G, Zohm H, Zucca C, 2010 Plasma Phys. Control. Fusion 52025002 
[28] Fevrier O, Maget P, Lutjens, Luciani J F, Decker J, Giruzzi G, Reich M, Beyer P, Lazzaro E, Nowak S, and the ASDEX Upgrade team 2016 Plasma Phys. Control. Fusion 58, 045015

[29] Fevrier O, Maget P, Lutjens H, Beyev P 2017 Plasma Physics and Controlled Fusion 59044002

[30] Gantenbein G, Zohm H, Giruzzi G, Gnter S,Leuterer F, Maraschek M, Meskat J, Yu Q, ASDEX Upgrade Team, and ECRH-Group (AUG) 2000 Phys. Rev. Lett. 851242

[31] Manini A, Berrino J, Cirant S, D’Antona G, Gandini F, Grunwald G,Leuterer F, Maraschek M, Monaco F, Neu G, Raupp G, Sormani D, Stober J, Suttrop W, Treutterer W, Wagner D, and Zohm H." Development of a feedback system to control MHD instabilities in ASDEX Upgrade". Fusion Engineering and Design, 82(5-14):995 1001, 2007. Proceedings of the 24th Symposium on Fusion Technology - SOFT-24.

[32] Zohm H, Gantenbein G, Giruzzi G, Gnter S,Leuterer F, Maraschek M, Meskat J, Peeters A G, Suttrop W, Wagner D, Zabiego M, ASDEX Upgrade Team, and ECRH Group et al 1999 Nucl. Fusion 30577

[33] Isayama A, Kamada Y, Ide S, Hamamatsu K, Oikawa T, Suzuki T, Neyatani Y,Ozeki T, Kajiwara K, Ikeda Y, and the JT-60 team 2000 Plasma Phys. Control. Fusion 42 L37

[34] Berrino J, Cirant S, Gandini F, Granucci G, Lazzaro E,Jannone F, Smeulders P, and DAntona G 2006 IEEE Trans. Nucl. Sci. 531009

[35] Petty C C, La Haye R J, Luce T C,Humphreys D A, Hyatt A W,Lohr J, Prater R, Strait E J and Wade M R. 2004 Nucl. Fusion 44243

[36] Volpe F A G, Austin M E, La Haye R J, Lohr J, Prater R,Strait E J, and Welander A S 2009 Phys. Plasmas 16102502

[37] Seol J C,Lee S G,Park B H, et al 2012 Phys. Rev. Lett 109, 195003

[38] Felici F, Goodman T P, Sauter O et al 2012 Nucl. Fusion 52074001

[39] Wang S, Ma Z W, and Zhang W. 2016 Phys. Plasmas 23052503

[40] Prater R, La Haye R J, Lohr J, Luce T C, Petty C C, Ferron J R, Humphreys D A, Strait E J, Perkins F W, and Harvey R W 2003 Nucl. Fusion 431128

[41] Hender T C et al., 2007 Nucl. Fusion 47 S128S202

[42] Hennen B A, Westerhof E,Nuij P W J M. et al 2010 Plasma Phys. Control. Fusion, 52104006

[43] van den Brand H et al 2012 Plasma Phys. Control. Fusion 54094003

[44] van den Brand H et al 2013 Nucl. Fusion 53013005 
[45] Mikhailovskii A B et al. 2008 Plasma Phys. Rep. 34538 and Mikhailovskii A. B et al. 2007 Phys. Plasmas 14112104

[46] Brunetti D, Lazzaro E, Nowak S, Sauter O, Graves J P, 2016 Journal of Physics: Conference Series 775012002

[47] Ramponi G, Lazzaro E and Nowak S, 1999 Phys. Plasmas 63561

[48] Sauter O et al.,2002 Plasma Phys. Control. Fusion 441999

[49] Hegna C C and Callen J D 1997 Phys. Plasmas 42940

[50] Sauter O et al 1997 Phys. Plasmas 41654

[51] Zohm H 1997 Phys. Plasmas 43432

[52] Giruzzi G, Zabiego M,Gianakon T A, Garbet X, Cardinali A, and Bernabei S 1999 Nucl. Fusion 39107

[53] Volpe F, Austin M E, Campbell G, and Deterly T, 2012 Review of Scientific Instruments 83, 103507

[54] Sauter O, Henderson M A,Ramponi G, Zohm H,Zucca C 2010 Plasma Physics and Controlled Fusion 52, 025002

[55] Fitzpatrick R and Hender T C 1994 Phys. Plasmas 113337

[56] Fitzpatrick R 1995 Phys. Plasmas 2825

[57] La Haye R J 2006 Phys. Plasmas 13055501

[58] Hahm T S and Kulsrud R M 1985 Phys. Fluids 28, 2412

[59] Fitzpatrick R 2003 Phys. Plasmas 102304.

[60] Salzedas F and JET EFDA Contributors 2011 Phys. Plasmas 18100701

[61] Sozzi C, Galpert G,Alessi E, et al 2015 Nucl. Fusion 55083010

[62] Ren C,Callen JD, Gianakon T A, et al,1998 Phys. Plasmas 5, 450

[63] Reich M, Bock A, Maraschek M, and ASDEX Upgrade Team.2012 FUSION SCIENCE AND TECHNOLOGY,61 309-313.

[64] Prater R, Farina D, Gribov Yu, Harvey R W,. Ram A K, Lin-Liu Y-R, Poli E, Smirnov A P, Volpe F, Westerhof E, Zvonkov A, and the ITPA Steady State Operation Topical Group. 2008 Nuclear Fusion, 48035006

[65] LaSalle J P Time optimal control systems 1959 Proc. Natl. Acad. Sci. USA 43 , 573577

[66] Kalman R E 1960 "On a General Theory of Control Systems" Proceed of the first IFAC Congress 481-491 
[67] Felici F,Rossel J X, Canal G et al EPJ Web of Conferences 20123202005

[68] Canal G P et al 2013 Nucl. Fusion 53113026

[69] Grasso D, Lazzaro E, Borgogno D and Comisso L 2016 Journal of Plasma Physics, 82 82, 595820603

[70] Lazzaro E,Nowak S,Sauter O et al, 2015 Nucl. Fusion 55, 093031.

[71] KasparekW, Petelin M, Shchegolkov ., Erckmann V, Plaum B, Bruschi A, at IPP Greifswald, E. G., Karlsruhe, F., and Stuttgart, I. 2008 Nuclear Fusion 48054010.

[72] Kasparek et al., Nucl. Fusion 57 (2016) 126001. 

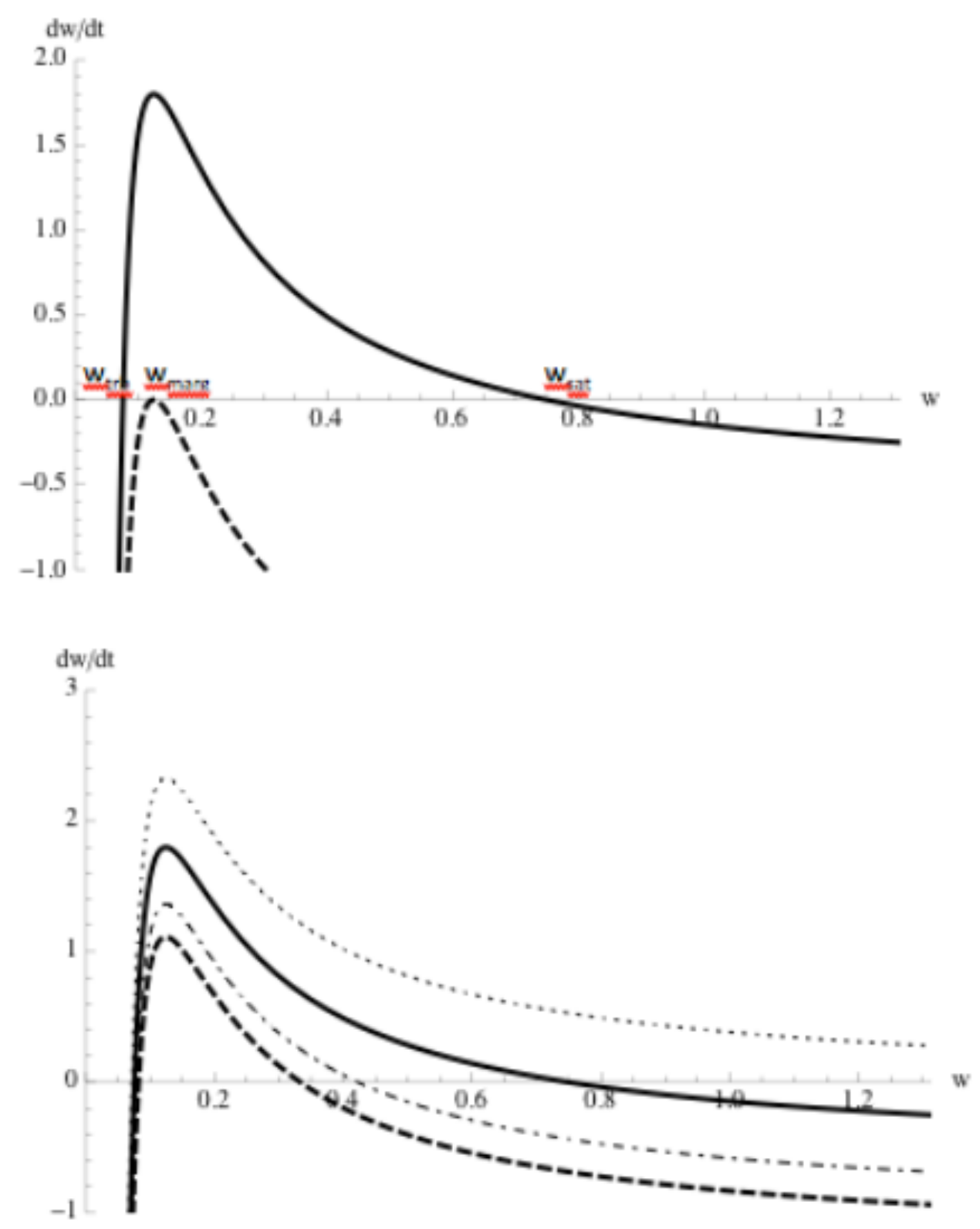

FIG. 1. (top) Sketch of dimensionless neoclassical growth rate $d w / d t$ vs $w(m=2)$ with indication of nomenclature $w_{\text {th }}, w_{\text {marg }}, w_{\text {sat }}$; an NTM island squeezed by ECCD below $w_{\text {marg }}$ is self extinguishing;(bottom) Neoclassical growth rate $d w / d t v$ vs $w$ for NTM modes (m=2) parameterised in terms of the ratio $y$ (flow shear/magnetic shear):(dots) $y=0.6$,(full) $y=0$,(dotdash) $y=1.1$, (dash) $y=1.4$. The shear ratio y hardly affects the threshold and marginal values of the island width while the saturation width may change considerably. 


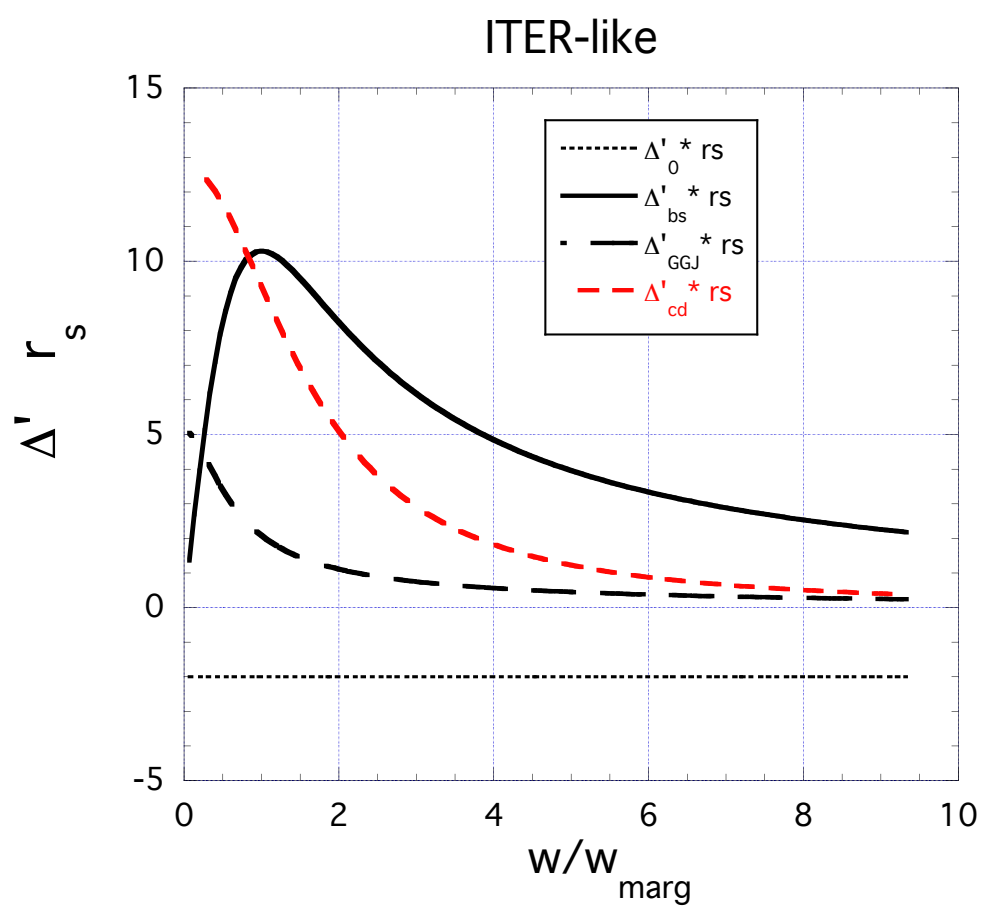

FIG. 2. Terms in the Rutherford equation (9) vs. $w / w_{\text {marg }}$ for $m=2, n=1$ NTM in ITER-like scenarios. Classical $\Delta_{0 J}^{\prime}$ (dotted line); neoclassical bootstrap effect $\Delta_{b s J}^{\prime}$ (full line); toroidal $\Delta_{G G J}^{\prime}$ (long dashed line); helical driven current $\Delta_{C D}^{\prime}$ (short dashed line) [12]. 


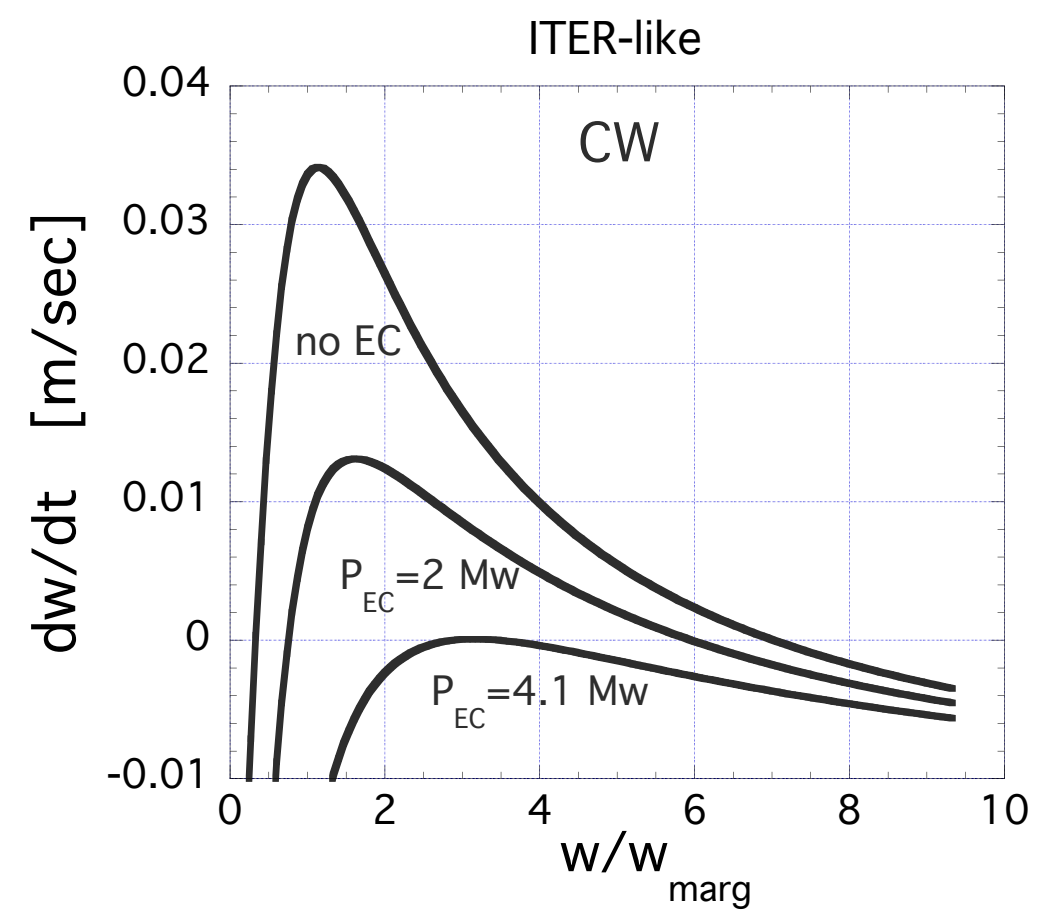

FIG. 3. NTM phase space $d w / d t$ vs. w/w $w_{\text {marg }}$ for an NTM 2/1. The effects of different values of $J_{C D}$ using $C W E C$ power injection for an ITER-like scenario are shown. The mode is suppressed by $4.1 \mathrm{MW}$ of EC power for $w_{c d}$ width $=0.04 \mathrm{~m}$. 


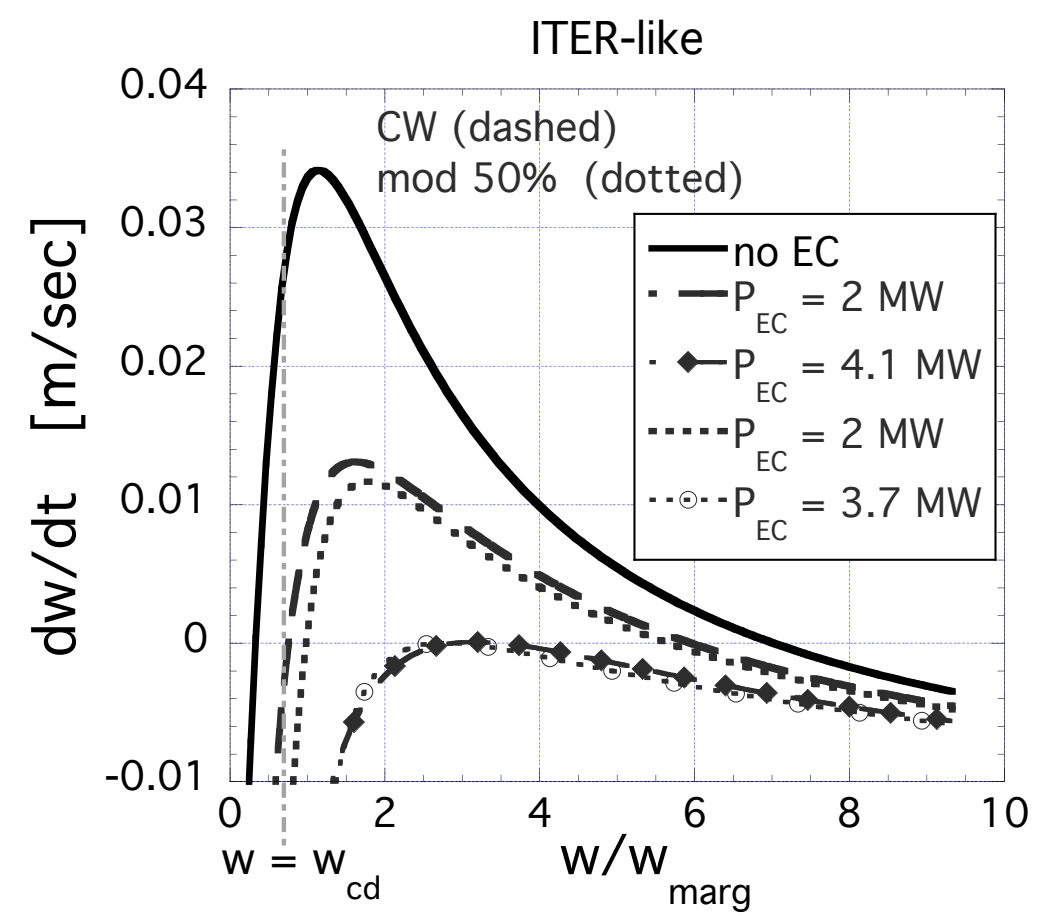

FIG. 4. NTM phase space $d w / d t$ vs $w$; example of control of $m=2, n=1$ NTM magnetic island width down to the self-extinguishing value, by perfectly phased $J_{C D}$ injection in an ITER-like scenario, showing the difference between $C W\left(0 \leq P_{E C}<4.1 M W\right)$ and $50 \%$ modulated case $C W\left(0 \leq P_{E C}<\right.$ $3.7 M W)$. 

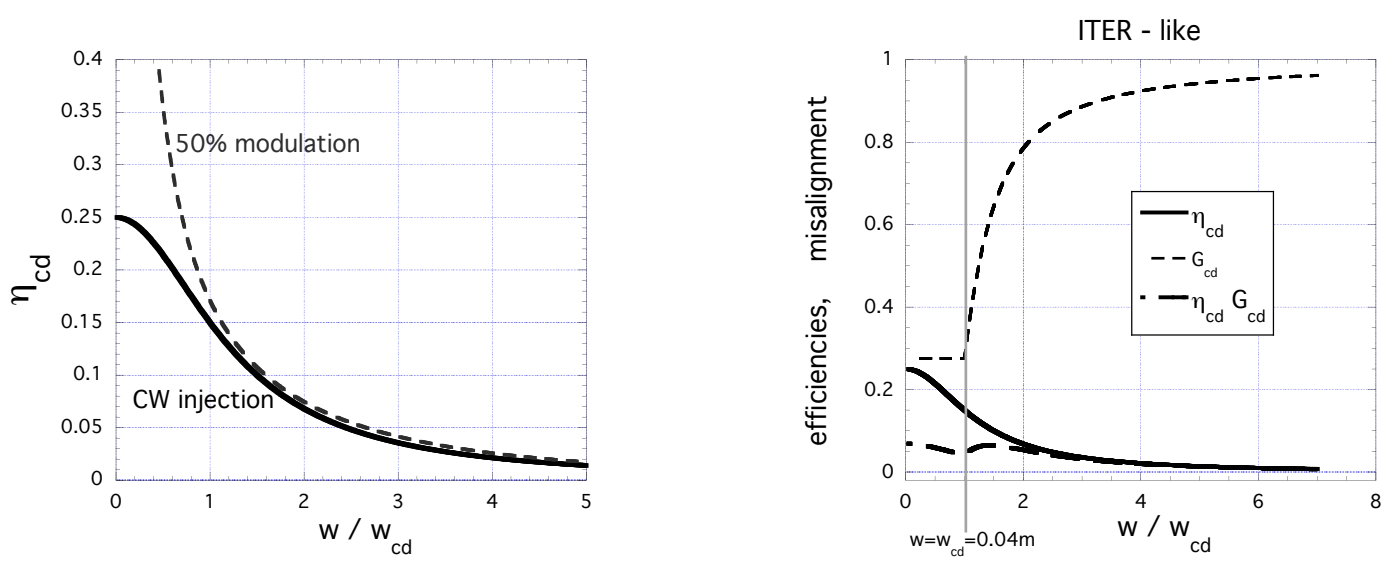

FIG. 5. (left) ECCD efficiency vs. $w / w_{c d}$ for $C W$ (full line) or $50 \%$ modulation (dashed line) of $J_{C D}$, perfectly phased and radially aligned on O-point [14] ; (right) plots of $\eta_{C D m . n}$ (full line)), $G_{C D}$ (thin-dashed line) and $\eta_{C D m . n} G_{C D} v s . w / w_{c d}$ (thick-dashed line) for a radial misalignment $\delta R=$ $0.016 m$ considering a 2/1 NTM. 

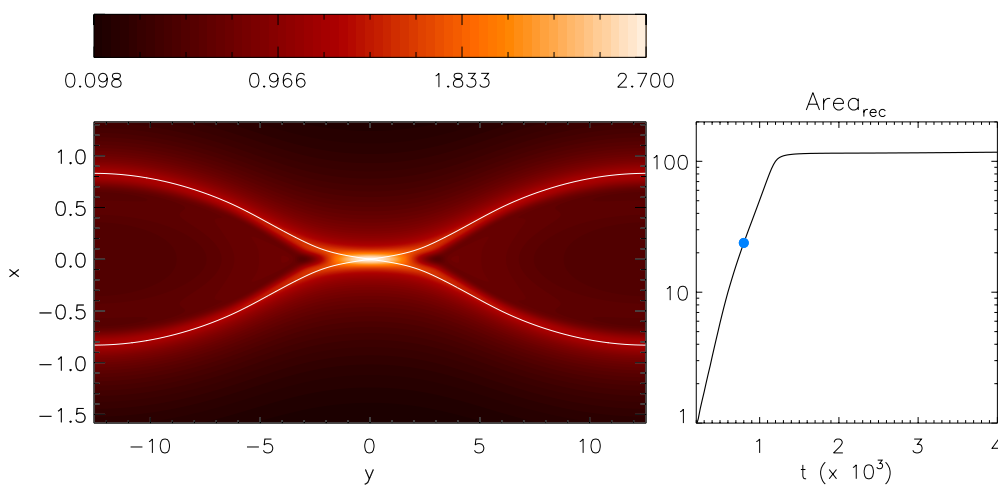

FIG. 6. Left frame. Contour plots of the current density at the time $t_{1}=800$, when the ECCD starts to be injected. The superimposed white lines identify the borders of the corresponding magnetic island. The rigth frame shows the time evolution of the magnetic island area in absence of ECCD control. 

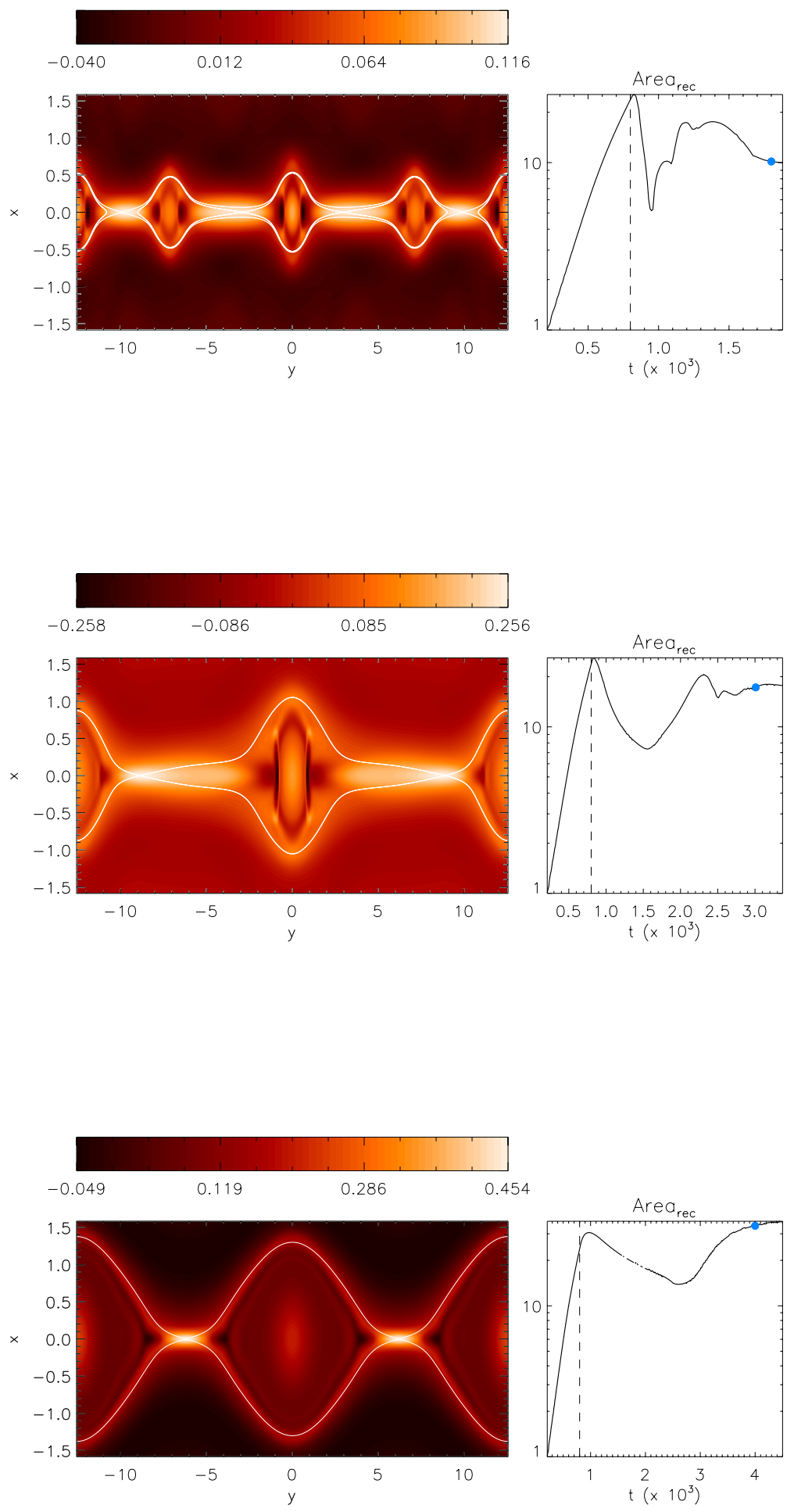

FIG. 7. Left column, from top to bottom. Contour plots of the current density in presence of $E C C D$ beams of width $w_{c d}=b\left[\psi_{X}-\psi_{O}\right]$ with $b=0.5,1,2$. The superimposed white lines identify the borders of the corresponding reconnected region. The rigth column shows the time evolution of the area of the reconnected region for the three cases. The dashed lines identify the starting injection time $t_{1}=800$, while the blue dots show the time when the current is plotted. 

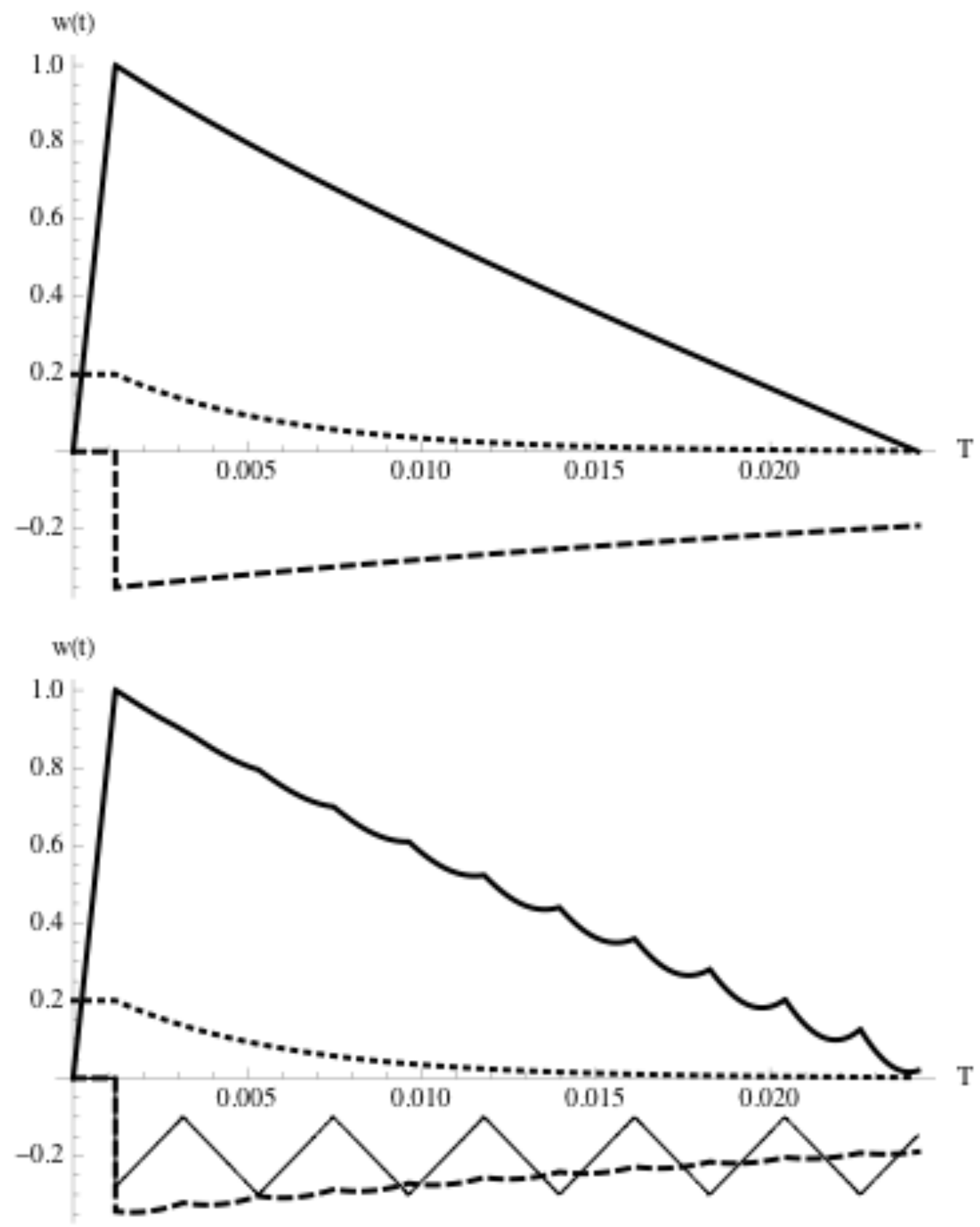

FIG. 8. Result of Optimal Control. (top)-a) (full line) suppression in given time $T$ of the incipient island width (normalzed at start of the control at $\left.t=1 / a_{11} ; b\right)$ (dotted) evolution of the frequency (normalized);c) (dashed) evolution of the control function u(t) (in a.u.) for fixed, small, radial misalignment ); (bottom)-Result of "Piecewise Optimal Control". a) (full line) Suppression in given time $T$ of the island width with intermittent sweeping of rational surface;b) (dotted) evolution of the frequency;c) (thin full line) offset waveform of the radial displacement $(t) ; d)$ (dashed) evolution of the resulting control function u(t) (in a.u.). 

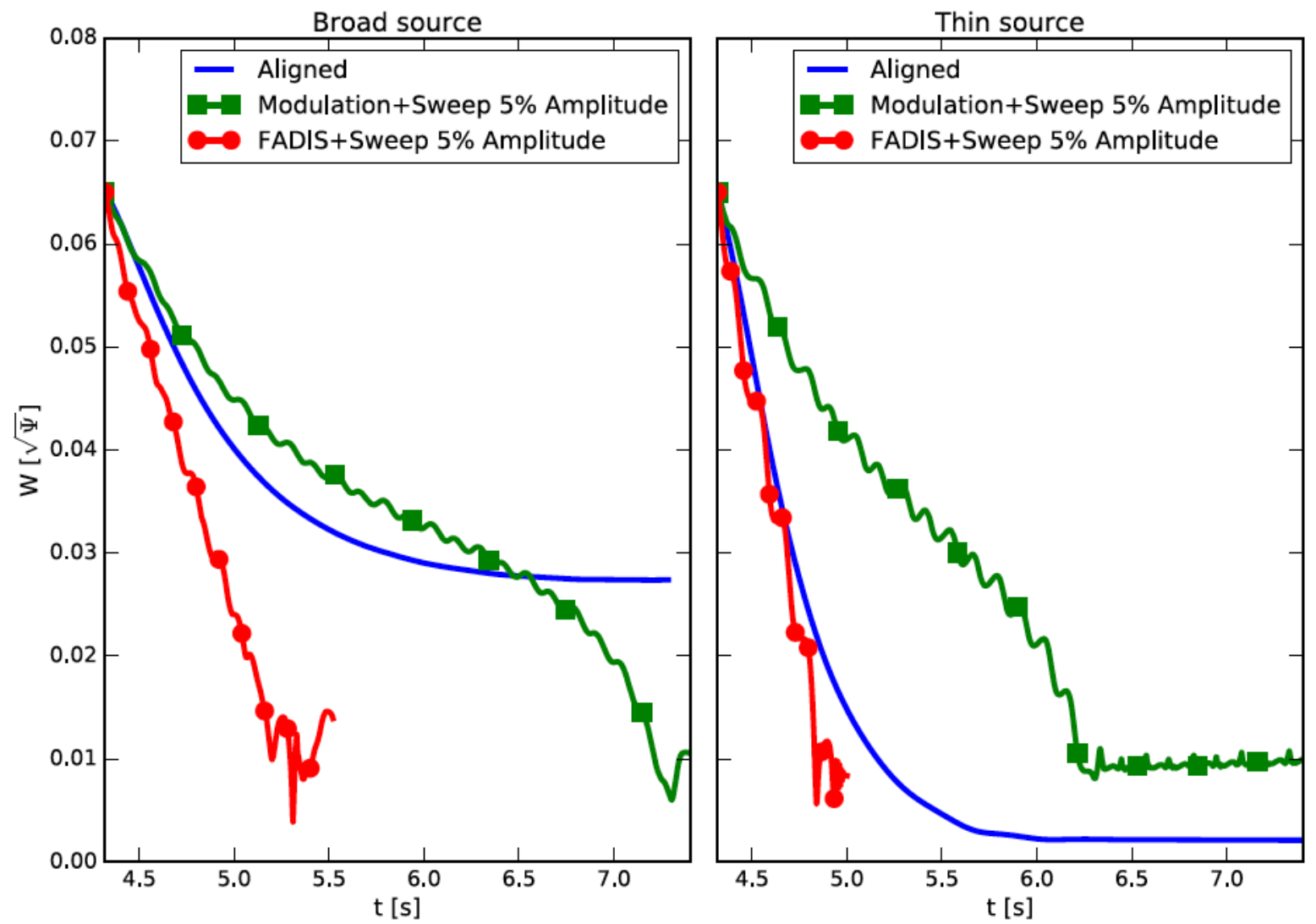

FIG. 9. Results of XTOR modelling by [29] of island stabilization by combined methods (modulation + rf beam sweeping or FADIS + rf beam sweeping ); In both cases, the island can be suppressed or drastically reduced, proving that these schemes are robust towards misalignment or deposition width uncertainties [O.Fevrier, P.Maget, H.Lutjens, P.Beyev,Plasma Physics and Controlled Fusion, 59:044002, 2017] 
Marginal Power for Preemption

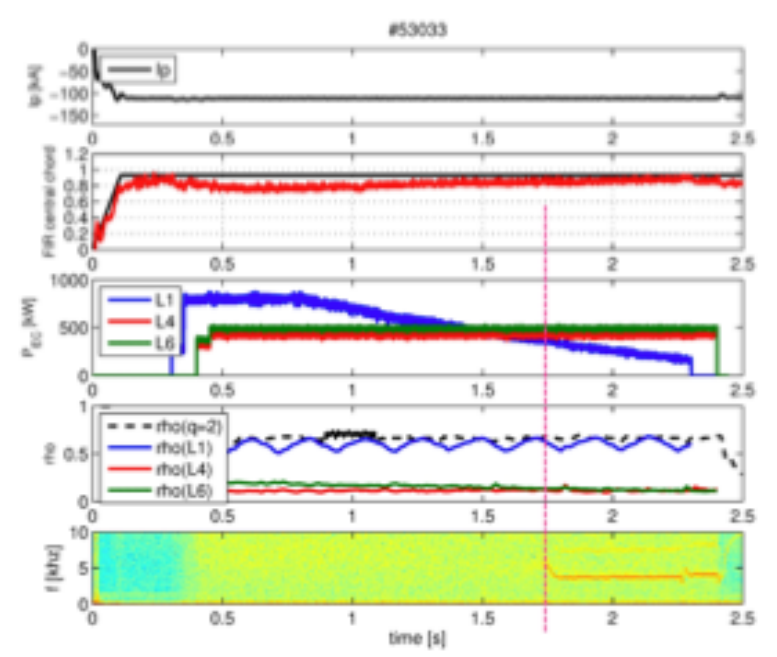

Full Preemption

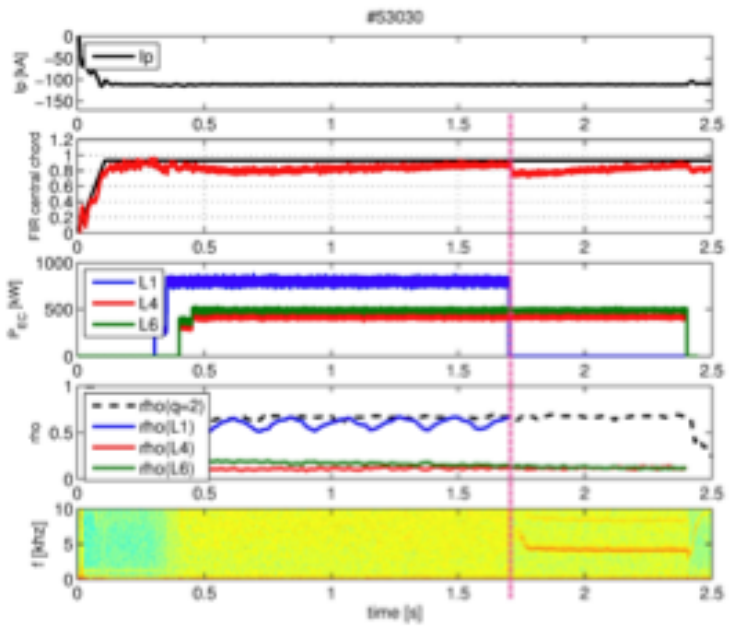

FIG. 10. Robust NTM control on TCV by intermittent sweeping of q-rational surface.From top to bottom: time traces of plasma current $I_{p}$, FIR signal, $P_{E C}(k W)$ of Gyroltrons $L_{1}, L_{4}, L_{6}$, sweeping waveform of (normalized) deposition radius $\rho$, spectrogram of modes (khz). In the left frame, the marginal power for pre-emption is found at $t \sim 1.75$ s. In the right frame, full stabilization is achieved with $P_{E C}$ larger than marginal value sweeping across the rational-q surface. 

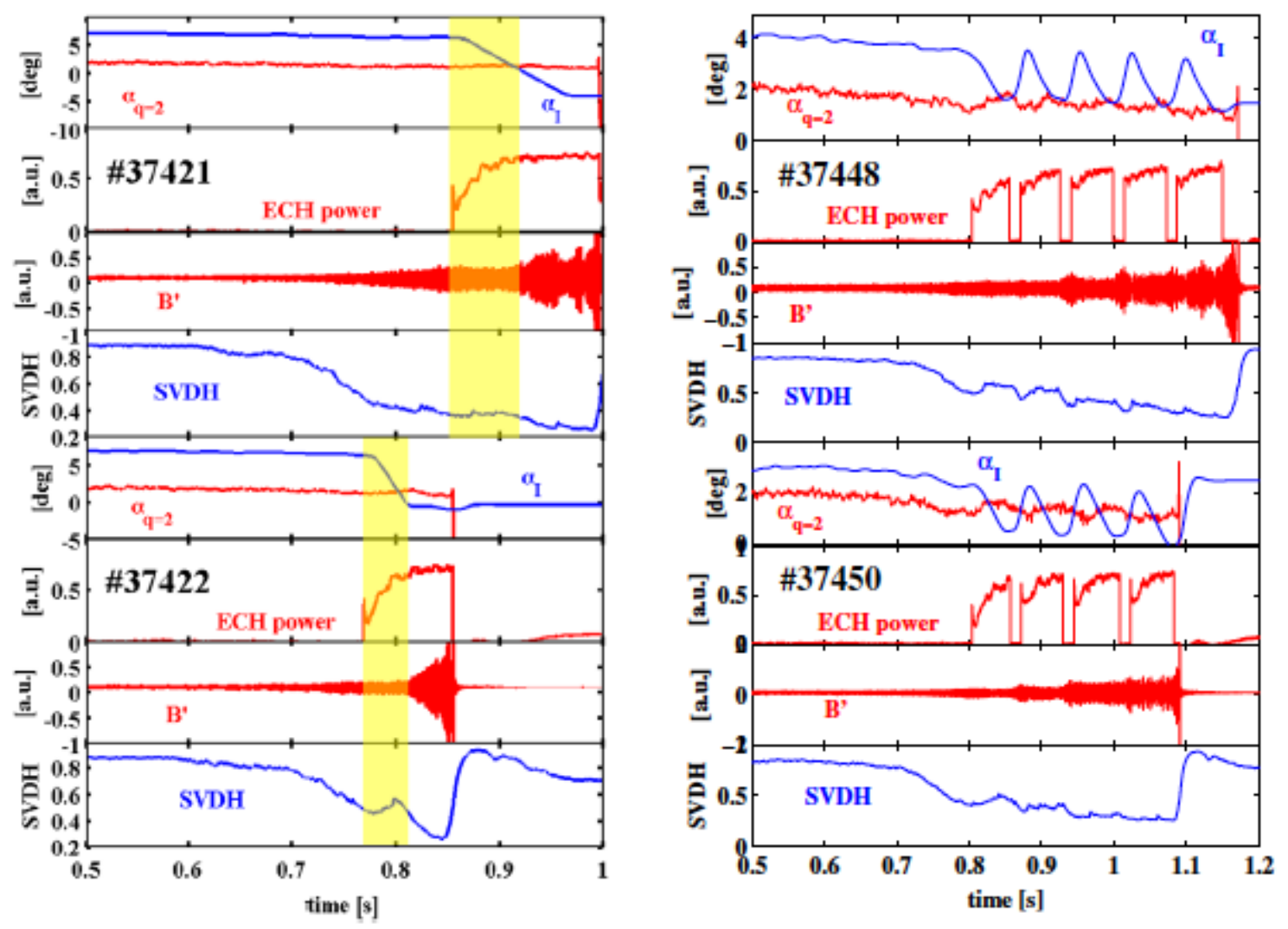

FIG. 11. Time evolution of the main $r$-t signals available during the MHD control experiment. A poloidal scan of the ECRH deposition is performed around the 2,1 island region. From top to bottom for each shot: RT reference angles of the poloidal injection of ECRH (0: horizontal, negative: inboard) and of the $q=2$ surface; ECRH power (in a.u.); pick-up coil signal (a.u.); SVDH marker. In these pulsed scans the ECRH power is switched off moving outward and switched on moving inward. The MHD oscillations appear depressed by ECRH pulses [C.Sozzi, G. Galperti,E. Alessi, et al,Nucl. Fusion 55083010 (2015)]. 